\title{
Index of Passages
}

Abbreviations are given according to the Thesaurus Linguae Latinae for Latin authors and according to Diccionario Griego-Español for the Greek ones. Only two exceptions are registered:

1. Florus' historiographical work is quoted following Malcovati $1972^{2}$.

2. Seneca's Historiae transmitted by P.Herc.1067 are here quoted per "cr" (cornice) and "pz" (pezzo) given the absence of a division in books and/or paragraphs in the edition by Piano in CErc 47 (2017), 163-250.

3. Only in Rich 'report Appianus' Bellum Civile is abbreviated E. (Emphylia) by declared author's will.

Anthologia Latina

409 R 9-12

166 n.111

\section{Ammianus Marcellinus Antiochenus}

14.6

14.6 .4

145 n.11

176 n.161

\section{Appianus}

Gall.

1.1-4

$1.2-11$

1.5-13

12-22

$B C$ (E.)

1.2

1.2.4-6

1.4-1.6

1.5.20-21

1.6.25

1.7-2.30

1.10.21

1.18-47

1.21

1.27

1.60.269

2.1-3.12

2.1.4

2.5-6

2.9 .18

2.9.21

2.22-35

2.31-5.145

3.13-16

3.21-4.8
338

338

338

338

147 n.18

334

335

335 n.17

335 n.17

338

335

335

334 n. 15

334 n. 15

335 n.16

335

335

336

336

336

336

338

335

336

\subsection{7-18}

4.11

336

4.110 .463

336

5.45 .191

5.145 .602

33.150

Hisp.

4-38

39-63

64-102

102

III.

4-5

7-8

9

10-11

11

13-30

14.42

30

Pun.

67-135

136

Mac.

1-4

4-19

Mith.

3-7

10-120

121

Praef.

6-7.19-24 335 n.18

$9.34 \quad 335 \mathrm{n} .18$

$11.44 \quad 336$ n.18 


$\begin{array}{ll}12.45-13.49 & 336 \\ 14.60 & 335 \text { n.17 } \\ \text { Sic. } & \\ 1-5 & 338 \\ 6-7 & 338 \\ & \\ \text { Syr. } & \\ 1-47 & 338 \\ 1.44 & 339 \\ 11-12 & 340 \\ 40-41 & 340 \\ 48-51 & 338\end{array}$

Aristoteles

Rh. 3.9.1409a-1409b 297 n.11

Arrianus

4.9.4

166 n.94

Aurelius Augustinus, episcopus Hipponensis

civ.

1.30

147 n. 18

2.18

147 n. 18

2.21

147 n. 18

Ps. Aurelius Victor Afer, Sex. epit. 2.3

78 n. 10

\section{de bello Alexandrino}

Alex.

58.4

166 n.112

de bello Hispaniensi

Bell. Hisp.

2

33

34

167 n.112

167 n. 112

167 n. .112

Calpurnius Flaccus

decl.

10.3

136 n.61

Cassiodorus, Flavius Magnus Aurelius chron.

2.161

238
Caesar, Caius Iulius

civ.

2.19

166 n.112

3.68 .1

178 n.168

Gall.

3.29.3 205 n.20

5.28.1 205 n.20

Cicero, Marcus Tullius

Arch.

$10.26 \quad 166$ n.110; 189 n.221

Att.

1.19.2 79 n.15

3.24.2 $211 \mathrm{n} .42$

14.14.2-3 289

16.4.2 $166 \mathrm{n} .111$

Brut.

$2.8 \quad 326$

$38 \quad 309$ n.54

$42 \quad 163 \mathrm{n} .100$

$132 \quad 309$ n.54

$274 \quad 309$ n.54

Cael.

$76.10 \quad 325$

Cato

$33 \quad 326$

$71.13 \quad 327$ n. 35

Cluent.

1207

de orat.

$1.157 \quad 310$ n.56

2.54186 n.207; 188 n.216;

189 n. 221

$2.64 \quad 300$ n.23

$2.95 \quad 309$ n.54

$3.10 \quad 210$

fam.

$2.13 .2 \quad 211 \mathrm{n} .42$

5.12.2 11 n.10; 349 n.72; 349

n.73

7.18.1 79 n.15

9.13.1 167 n.112

$15.19 \quad 165$ n.97

fin.

$2.74 \quad 209$ n.30

5.85.11-12 $\quad 45 \mathrm{n} .49$ 
leg.

$1.5-9$
leg. agr.
3.5
Manil.
28
off.
1.26
1.112
orat.
30.107
$62-64$
64
66

Phil.

3.2

3.7

3.39

Planc.

76

prov.

19

32

35-36

47

p. red. in sen.

8

rep.

1.27-28

2. 11

2. 14-15

2.23

2.43-44

3.43

S. Rosc.

2.51

Tusc.

2.5

Vatin.

23

Verr.

I.125

II.2.3-4
302 n. 33

289

349 n.72

287 n. $48 ; 288$

289

325 n. 33

300 n. 24

307 n. $49 ; 310$ n. 55

186 n. 207; 188 n.216;

189 n.221; 300 n. 25;

305 n.40

209 n. 30

209 n. 30

209 n. 30

211 n. 42

79 n.15

79 n.15

79 n. 15

79 n.15

209 n.30

289 n. 52

104 n.13

277 n.2

289 n. 52

289 n. 52

289

290

109

289

209 n. 30

36 n.22
Curtius, Rufus Quinctus

$\begin{array}{ll}8.1 .45 & 163 \mathrm{n} .94 \\ 8.5 .13 & 163 \mathrm{n} .94 \\ 10.3 .9 & 117 \mathrm{n} .61 \\ 10.5 .10 & 167 \mathrm{n} .118\end{array}$

Demetrius, rhetor

Eloc.

1-35 297

10-11 297 n.13

10-22 297

$11 \quad 297$ n.14; 300 n.22

12-13 299 n.20

$14 \quad 299$ n.18

19-20 301 n.28

19-21 298 n.17

$33 \quad 299$ n.18

$108 \quad 299$ n.18

128-189 297

190-239 297

240-304 297

Demostenes

In epist. Phil.

13

163 n.95

Olynth.

$2.20 \quad 163$ n.65

\section{Dio Cassius}

$\begin{array}{ll}42.15 & 185 \mathrm{n} .201 \\ 43.25 .1-2 & 131 \\ 43.29 .3 & 167 \mathrm{n} .112 \\ 43.32 .3 & 167 \mathrm{n} .112 \\ 43.39 .1 & 166 \mathrm{n} .111 \\ 45.10 .1 & 166 \mathrm{n} .111 \\ 53.19 & 251 \\ 55.13 & 131 \mathrm{n} .35 \\ 55.13 .4 & 132 \mathrm{n} .40 \\ 57.7 .1 & 276 \\ 57.7 .6 & 126 \mathrm{n} .14 ; 244 \mathrm{n} .47 \\ 57.8 .1-2 & 84 \mathrm{n} .50 \\ 57.13 .6 & 276 \\ 57.15 .2 & 126 \mathrm{n} .15 \\ 57.15 .3-4 & 126 \mathrm{n} .18 \\ 57.15 .8 & 126 \mathrm{n} .14 \\ 57.18 .10 & 80 \mathrm{n} .25 \\ 57.18 .11 & 263\end{array}$




\begin{tabular}{|c|c|c|c|}
\hline \multirow{2}{*}{$\begin{array}{l}57.19 .1 \\
57.19 .6\end{array}$} & \multirow{2}{*}{$\begin{array}{l}276 \\
127\end{array}$} & \multicolumn{2}{|c|}{ Florus, Lucius Annaeus } \\
\hline & & \multicolumn{2}{|l|}{ epit. } \\
\hline $57.19 .7-8$ & 276 & \multirow[t]{2}{*}{ 1.praef. $4-8$} & 118 n.63; 145 n.11; \\
\hline $57.20 .1-2$ & 126 n.18 & & 176 n.161; 336 n.23 \\
\hline $57.24 .2-4$ & 153 n.42 & 1.praef. 5 & 327 \\
\hline 58.2 .1 & 126 n.14 & 1.praef. 5-8 & 335 n.18 \\
\hline 58.4 .3 & 126 n.18 & 1 praef. $7-8$ & 323 \\
\hline 58.21 .5 & $126 \mathrm{n} .14$ & 1 praef. 7 & 325 n.31 \\
\hline $58.24 .3-4$ & 83 n.46 & 1 praef. 8 & 91; 176 n.161; 25 n.31 \\
\hline $58.24 .3-5$ & 155 n.42 & $1.1-3$ & 335 n.19 \\
\hline $59.9 .3-4$ & 140 & 1.2 & 119 \\
\hline $59.3 .5-6$ & 139 & $1.4-16$ & 335 \\
\hline 59.9 .5 & 132 n.40 & 1.17 & $119 ; 325$ n.19 \\
\hline 59.24 .4 & 128 n.26; 149 n.24 & 1.18 & 319 n.16; 336 n.23 \\
\hline 59.27 .3 & 141 n.76 & $1.18 .1(1)$ & 324 n.30 \\
\hline 61.1 .1 & 274 n.47 & 1.18 .2 & 178 n.168 \\
\hline 64.21 & 163 n.92 & $1.18-47$ & 335 \\
\hline \multicolumn{2}{|l|}{ Epit.Xiph. } & 1.19 & 147 n.18; 320 \\
\hline \multirow[t]{2}{*}{ 57. $20.2-4$} & \multirow[t]{2}{*}{244} & 1.19 .2 & 178 n.168 \\
\hline & & $1.31 .1-6$ & 176 n.161 \\
\hline \multicolumn{2}{|c|}{ Diodorus Siculus } & 1.34 & 119 n.67; 120 n.67; \\
\hline 11.9 .4 & 163 n.96 & & $\begin{array}{l}335 \text { n.18; } 336 \text { n.20; } \\
336 \text { n.23 }\end{array}$ \\
\hline \multicolumn{2}{|c|}{ Diogenes Laertius } & 1.34 .19 & 191 n.227; 320 \\
\hline 9.10 .60 & 163 n.93 & 1.47 & $\begin{array}{l}\text { 118; 119; } 120 ; \\
320 ; 335 \text { n.18; } 336\end{array}$ \\
\hline \multicolumn{3}{|c|}{ Dionysius Halicarnassensis } & n. $20 ; 336$ n.23 \\
\hline $5.77 .4-5$ & 288 n.50 & $1.47 .1-3$ & 119 n.67 \\
\hline \multirow[t]{2}{*}{22.148} & \multirow[t]{2}{*}{299 n.18 } & 1.47 .1 & 120 n.68 \\
\hline & & 1.47 .2 & 147 n.18 \\
\hline \multicolumn{2}{|c|}{ Ennius, Quinctus } & 1.47 .3 & 320 \\
\hline ann. $24 \mathrm{~S}$ & 209 n.31 & 1.47 .6 & 120 n.68; 320 \\
\hline \multirow{2}{*}{\multicolumn{2}{|c|}{ trag. frg. 381-382 Ribbeck 287 n.48 }} & $1.47 .7-8$ & 98 n.28 \\
\hline & & $1.47 .7-13$ & 120 \\
\hline \multicolumn{2}{|l|}{ Epicurus } & 1.47 .7 & 117 n.61; 176 n.161 \\
\hline \multicolumn{2}{|l|}{ nat. } & $1.47 .8-14$ & 321 \\
\hline \multirow[t]{2}{*}{21} & \multirow[t]{2}{*}{57 n.29 } & 1.47 .8 & 121 \\
\hline & & 1.47.11 & 121 \\
\hline \multicolumn{2}{|l|}{ Euripides } & 1.47 .12 & 121; 191 n.227 \\
\hline Phoen. & & 1.47 .13 & 121 n.70 \\
\hline 393 & 83 n.46 & 2.1 & 135 n.57; 191; 336 \\
\hline & & & n.20 \\
\hline Flavius lose & & $2.1-2$ & 147 n.18 \\
\hline Al & & $2.1-4$ & 335 \\
\hline 19.167-180 & 278 & 2.2 & 191 \\
\hline 18.101-102 & 141 & 2.3 & 191 \\
\hline & & 2.4 & 191 \\
\hline
\end{tabular}




\begin{tabular}{|c|c|c|c|}
\hline $2.5-6$ & 336 & I 506-507 & 68 n.76; 103 n.8; 127 \\
\hline 2.5 & $191 ; 135$ n.58 & & n.19; 345 n.51 \\
\hline 2.6 & 191 & $1506-508$ & 260 n. $4 ; 346$ n.56 \\
\hline $2.7-8$ & 336 n.21 & 1507 & 129 n.26; 129 n.29; \\
\hline $2.9-18$ & 336 & & 316 \\
\hline 2.13 & 118 & I 507-508 & 104 n.16 \\
\hline 2.13 .1 & 117 n.60 & 1511 & 23 n.52 \\
\hline $2.13 .8-17$ & 117 & 1518 & 239 n.15 \\
\hline 2.13 .8 & 117 & 1519 & 245 n.60 \\
\hline 2.13 .14 & 117 n.60 & I 520-521 & 238 n.13 \\
\hline 2.13.37-39 & 192 n.228 & 1520 & 238 n.11 \\
\hline 2.13 .50 & 192 n.228 & 1521 & 246 n.60 \\
\hline $2.13 .80-83$ & 192 n.228 & 1522 & 240 n.25 \\
\hline 2.13 .90 & 192 n.228 & 1523 & 239 n. $21 ; 255$ \\
\hline 2.14 .4 & 324 n.28 & I 525-586 & 281 n.16 \\
\hline 2.14 .5 & 324 & 1530 & 245 n.58 \\
\hline 2.17 .1 & 322 n.21 & 1597 & 95 n.23; 319 n.13; 322 \\
\hline 2.17 .10 & 322 n.21 & & n.21 \\
\hline $2.19-20$ & 191 & II 982-985 & 77 n.6 \\
\hline $2.22-34$ & 336 & III 533-534 & 205 n.18 \\
\hline 2.34 & 335 n.18 & III 572 & 209 n.30 \\
\hline $2.34 .64-65$ & 324 & III 596-597 & $\begin{array}{l}77 \text { n.6; } 77 \text { n.8; } 104 \\
\text { n.16; } 195\end{array}$ \\
\hline \multicolumn{2}{|c|}{ The Fragments of Roman Historians } & III 596 & 77 n.7; 249 n.85; 345 \\
\hline \multicolumn{2}{|l|}{ FRHist. } & & n.51 \\
\hline 138 & 202 n.8 & $26 \mathrm{~T} 13$ & 349 n.75 \\
\hline | 118-119 & 77 n.6 & $30 \mathrm{~T} 1$ & 11 n.10; 349 n.73 \\
\hline 1296 & 12 n.15 & 46 & $21 ; 27$ \\
\hline 1308 & 349 n.73 & 47 & $21 ; 27$ \\
\hline 1312 & 349 n.73 & 48 & $20 ; 27$ \\
\hline 1365 & 12 n.14 & 49 & $21 ; 27$ \\
\hline $448-450$ & 205 n.17 & 50 & $20 ; 27$ \\
\hline I 489-496 & 135 n.54 & 53 & $21 ; 27$ \\
\hline 1449 & 14 n.25 & 54 & $20 ; 27$ \\
\hline | 475-481 & 13 n.20 & 56 & $19 ; 27 ; 200$ \\
\hline 1478 & 14 n.22 & $56 \mathrm{F9}$ & 25 \\
\hline 1483 & 14 n.26 & 57 & $21 ; 27$ \\
\hline I 489-490 & 15 n.27 & 58 & $14 ; 27 ; 200 ; 204$ \\
\hline I 492 & 15 n.29 & 59 & $21 ; 27$ \\
\hline 1499 & 246 n.66 & $59-69$ & 200 \\
\hline 1505 & 88 n.2 & 60 & 27 \\
\hline I 505-508 & 195 & 61 & $20 ; 28$ \\
\hline \multirow[t]{4}{*}{1506} & 9 n.3; 10 n.6; 77 n.8; & 62 & 28 \\
\hline & 103 n.9; 127 n.19; & $62 \mathrm{~T} 2$ & $19 \mathrm{n} .41$ \\
\hline & 195; 218 n.24; 316 & 63 & 28 \\
\hline & n.6; 349 n.74 & 64 & $14 ; 28$ \\
\hline I 506 n.18 & 348 n.71 & $64 \mathrm{~F} 3$ & 14 \\
\hline
\end{tabular}




$\begin{array}{ll}64 \mathrm{~F} 4 & 14 \\ 65 & 21 \\ 66 & 21 \\ 67 & 21 \\ 68 & 21 \\ 70 & 15 ; 28 ; 200 \\ 70 \mathrm{~F} 8 & 135 \mathrm{n} .54 \\ 70 \mathrm{~F} 11 & 135 \mathrm{n} .54 \\ 70 \mathrm{~F} 15 & 135 \mathrm{n} .54 \\ 70 \mathrm{~T} 2 & 15 ; 135 \mathrm{n} .5 \\ 71 & 19 ; 28 ; 200 ; 209 \\ 72 & 22 ; 28 ; 200 \\ 73 & 28 \\ 73-75 & 200 \\ 74 & 28 \\ 74 \mathrm{~F} 1 & 64 \mathrm{n} .60 ; 123 \\ 74 \mathrm{~F} 2 & 64 \mathrm{n} .60 \\ 74 \mathrm{~T} 1 & 343 \mathrm{n} .44 \\ 75 & 23 ; 28 \\ 76 \mathrm{~T} 1 & 14 \mathrm{n} .23 \\ 78 & 22 ; 200 ; 212 \\ 78 \mathrm{~T} 3 \mathrm{ab} & 66 \mathrm{n} .66 \\ 79 & 22 \\ 79 \mathrm{~F} 4 & 23 \\ 80 & 23 \\ 80 \mathrm{~F} 4 & 23 \\ 80 \mathrm{~T} 5 & 66 \mathrm{n} .66 \\ 80 \mathrm{~T} 51 & 66 \mathrm{n} .66 \\ & \end{array}$

2.3

145 n.11

3.1

106 n.23; 106 n.24

Comm.

19.2

281 n.17

Heliog.

$1.1284 \mathrm{n} .33$

$33.1 \quad 284 \mathrm{n} .33$

$34.1 \quad 284 \mathrm{n} .33$

Marcus

$28.10 \quad 281 \mathrm{n} .17$

M. Aur.

$28.10 \quad 284$ n.33

Horatius Flaccus, $\mathbf{Q}$.

ars

156-178 105

carm.

1.24.7 $173 \mathrm{n} .148$

2.1.1-2 $116 \mathrm{n} .58$

2.1.1-8 93; 343 n.37

$2.11 .5 \quad 175$ n.156

2.12.10 21

epist.

$1.3 \quad 119 \mathrm{n} .66$

$2.2 \quad 119 \mathrm{n} .66$

epod.

16.1-2 $114 \mathrm{n} .49$

sat.

$1.3 \quad 20$

$1.10 .86 \quad 20$

Fronto, M. Cornelius
ep. 9 (224.12 van den Hout) 79 n.15

Gellius, A.

$\begin{array}{ll}\text { 4.3 praef. } 2 & 136 \mathrm{n} .59 \\ 5.18 .1-2 & 11 \mathrm{n} .11 ; 93 \\ 10.11 .3 & 327 \\ 12.11 .7 & 173 \mathrm{n} .148 \\ 14.2 .1 & 132 \mathrm{n} .42\end{array}$

Incertus poeta

Laus luv.

$5.109 \quad 100$ n.37

Laus Mart.

4.40.1-2 $\quad 100$ n.37

$12.36 .8 \quad 100$ n. 37

Scriptores Historiae Augusta

Aurel.

42.3

290 n. 54

42.5

284 n. 33

Avid.

$8.3 \quad 284$ n. 33

Car.

2.1-3.2

105 n.17; 176 n.161

lustinus, M. Iunian(i)us

38.3.11

206

38.4.1-7.10 200

38.4-7 205

38.6.3-4 206

38.6.7 206 
Lactantius, L. Cae(ci)lius Firmianus

inst.

2.4.36-37

7.15.14-16

7.15 .14

7.15 .15

7.15 .16

165 n.99

$89 ; 145$ n. 8

64 n. $60 ; 128 ; 195$;

249; 252; 319 n.14;

344

120 n. 68

172 n.143

\section{Livius, T. Patavinus}

praef. 4

321 n.18

praef. 9-12

111 n. 41

praef. 9

1.38 .3

5.27 .2

7.10 .5

8.37 .8

23.5.12

27.20 .9

34.4.1-2

44.22.10

45.45 .5

operis Liviani integri periochae

Liv. perioch. 109 116; 116 n.58

\section{Lucanus, Marcus Annaeus}

1.1-32

1.8

$1.10-23$

1.67

1.67-182

1.67-69

1.67-97

1.68-70

$1.70-72$

$1.72-80$

1.81-82

1.82-84

1.82-157

1.109-111

1.125-126

1.158-161

1.158-182

1.159-182

1.159
154 n.51

116 n. .56

114 n. 50

96

113

113 n. 47

176 n.161

117 n. 60

96

176 n.161

$113 ; 114$

117 n.60

114; 117; 117 n.59

117 n. 60

117 n.60

121

114

178 n.164

115 n.53
1.161

115 n.53

1.162-164

121

1.163-164

116

1.164-165

116 n.55

1.165-166

1.166-167

1.173

1.173-174

1.173-182

1.175-176

1.177

1.179

2.139-236

2.140-141

2.145-147

2.148-151

2.160-161

2.171-173

2.173-187

2.207-209

2.220

2.221-224

9.204-205

116

116

99

121

142 n.18

115 n.53

99

121

281

281

282

282

282

282

147 n.18

282

282

282

281

Lucianus sophista

Hist. Cons.

48

331 n. 5

Lydus, Iohannes Laurentius

Mag.

2.6

132

Martialis, M. Valerius

1.61.7-8

77 n. $9 ; 150$ n. $28 ; 150$

n.29; 174 n.152

1.61 .7

$87 ; 150$ n.29

1.61 .8

150 n.29

3.12 .1

174 n. 150

3.63 .4

174 n. 150

4.40.1-2

77 n.9; 150 n.29

4.40 .2

$87 ; 150$ n.29

6.19

180 n.176

10.72.8-13

77 n.9

Orosius, Paulus (?)

hist.

5.8 .2

147 n.18 
Ovidius Naso, $\mathrm{P}$.

fast.

$\begin{array}{ll}1.21-22 & 265 \mathrm{n} .21 \\ 1.22-23 & 265 \\ 1.608 & 84 \mathrm{n} .49 \\ \text { met. } & \\ \begin{array}{l}15.199-213 \\ \text { Pont. }\end{array} & 327 \mathrm{n} .36 \\ 2.5 .53-56 & 265 \\ 4.10 & 266 \\ 4.16 .6 & 266 \mathrm{n} .24 \\ \text { trist. } & \\ 4.10 .17 & 167 \mathrm{n} .118 \\ 4.10 .23-26 & 189 \mathrm{n} .221\end{array}$

Petronius (Arbiter)

46

181 n.183

Plautus, T. Macc(i)us

Trin.

186

Merc.

790-791

136 n. 60

Plinius Secundus, C. (vulgo Plinius maior) nat.

praef. 20

66 n.66; 128 n.24;

180 n. $175 ; 238$

3.1 .10

7.84

14.5

19.90

24.43

28.29

33

33.30

33.30-34.30

33.146

33.148-150

166 n. 110

245 n. 59

133 n. 45

245 n.59

239 n.18

239 n.18

132

133

133; 133 n.43

135 n. 54

120 n.69

147 n. 18

33.150

239 n.18

37.81

Plinius Secundus, C. (vulgo Plinius minor) epist.

$\begin{array}{ll}1.13 .3 & 24 ; 239 \\ 3.5 .3 & 245 \text { n. } 54 \\ 3.5 .4 & 245\end{array}$

3.5 .6

66 n. $66 ; 238$

3.5.10

331 n. 5

3.16 .6

207

4.9

181 n.183

5.5 .3

240

5.8

186 n.207

8.12.4-5

240 n.22

paneg.

84.1

173 n.148

Plutarchus Cheronensis

Alex.

28

163 n.94

50

163 n.94

Apoth. Lac.

225 D $13 \quad 163 \mathrm{n} .96$

Cato

20

182 n.188

Polybius

2.38.5

178 n.169

Priscianus

GL

$2.248 .4 \quad 22 \mathrm{n} .46$

3. $410 \mathrm{~K}$

89

Scripta Probiana

vita Pers.

5

240 n. 25

Quintilianus, M. Fabius

decl.

$10.3 \quad 136 \mathrm{n} .61$

$251 \quad 136 \mathrm{n} .61$

$258.9 \quad 207$

$262 \quad 136 \mathrm{n} .61$

$327 \quad 136 \mathrm{n} .61$

$368 \quad 136 \mathrm{n} .61$

inst.

2.5.20 189 n.224

3.5.8 280

3.8.20 $79 \mathrm{n} .15$

3.8.46-47 280

$3.8 .46 \quad 286$

$3.8 .47 \quad 280$

$3.8 .53 \quad 280$ 


$\begin{array}{ll}5.10 .30 & 280 \\ 5.10 .71 & 280 \\ 7.4 .38 & 136 \mathrm{n} .61 \\ 8.2 .9 & 280 \\ 8.2 .18 & 189 \mathrm{n} .224 \\ 8.3 .32 & 289 \mathrm{n} .51 \\ 8.6 .32 & 289 \mathrm{n} .51 \\ 9.2 .42 & 89 \\ 9.2 .98 & 137 \mathrm{n} .64 \\ 9.4 .1 & 170 \mathrm{n} .33 \\ 10.1 .31 & 157 \mathrm{n} .61 ; 189 \mathrm{n} .221 \\ 10.1 .34 & 180 \mathrm{n} .176 \\ 10.1 .39 & 189 \mathrm{n} .224 \\ 10.1 .90 & 266 \mathrm{n} .24 \\ 10.1 .102 & 24 ; 239 ; 240 \\ 10.1 .103 & 212 \mathrm{n} .48 ; 238 \\ 10.1 .104 & 129 \mathrm{n} .30 ; 210 \\ 10.1 .105 & 170 \mathrm{n} .133 \\ 10.1 .129 & 145 \mathrm{n} .10 \\ 10.2 .17 & 204 \mathrm{n} .16 \\ 10.3 .7 & 186 \mathrm{n} .207 \\ 11.1 .17 & 170 \mathrm{n} .133 \\ 12.1 .14 & 170 \mathrm{n} .133 \\ 12.1 .16 & 170 \mathrm{n} .133 \\ 12.7 .10 & 181 \mathrm{n} .183 \\ 12.10 .12 & 170 \mathrm{n} .133 ; 309 \mathrm{n} .54\end{array}$

\section{Pseudus Quintilianus}

decl.

$\begin{array}{ll}3.7 & 207 \\ 253 & 279 \mathrm{n} .6 \\ 267 & 279 \mathrm{n} .6 \\ 269 & 279 \mathrm{n} .6 \\ 274 & 279 \mathrm{n} .6 \\ 282 & 279 \mathrm{n} .6 \\ 288 & 279 \mathrm{n} .6 \\ 293 & 279 \mathrm{n} .6 \\ 329 & 279 \mathrm{n} .6 \\ 345 & 279 \mathrm{n} .6 \\ 351 & 279 \mathrm{n} .6 \\ 352 & 279 \mathrm{n} .6 \\ 374 & 279 \mathrm{n} .6 \\ 382 & 279 \mathrm{n} .6\end{array}$

Rhetorica ad C. Herennium Rhet. Her.

2.40 .3

89 n.6

\section{Sallustius, C. Crispus}

Catil.

$\begin{array}{ll}1.7 .4 & 214 \mathrm{n} .60 \\ 2.101 .3 & 214 \mathrm{n} .60 \\ 4.2 & 159 \mathrm{n} .71 \\ 5.8 & 111 \mathrm{n} .38 \\ 8.1 & 178 \mathrm{n} .168 \\ 10-13 & 115\end{array}$

10.1

106 n.23; 176 n.160

10.2-3 $111 \mathrm{n} .38$

$11.1 \quad 157 \mathrm{n} .64$

12.1-2 $111 \mathrm{n} .38$

$12.2 \quad 110$ n.37

12.3-4 $112 \mathrm{n} .45$

13.3-5 110 n.37

$41.3 \quad 178 \mathrm{n} .168$

$51.25 \quad 178 \mathrm{n} .168$

$53.5 \quad 111 \mathrm{n} .38$

epist.

2.7.11 $\quad 134 \mathrm{n} .48$

2.12.1 $134 \mathrm{n} .48$

hist.

1.1-18 Maurenbrecher $\quad 348$

1.5 Maurenbrecher $\quad 157$ n.64

1.6 Maurenbrecher=1.12 La Penna/Funari 103 n.11

1.9 Maurenbrecher $\quad 145 \mathrm{n} .11$

1.11 Maurenbrecher=1.15 La Penna/Funari 107 n.26; 111 n. $39 ; 115$

n. $53 ; 145$

n. $11 ; 147$

n. $18 ; 176$

n.160

1.12 Maurenbrecher=1.16 La Penna/Funari 107 n. 26; 145 n.12; 147 n. $18 ; 176$ n.160

1.16 Maurenbrecher=1.17 La Penna/Funari 111 n. $39 ; 145$ n. $16 ; 157$ n. $64 ; 176$ n. 160

1.18 Maurenbrecher=1.20 La Penna/Funari 115 n.53

1.19-53 Maurenbrecher 348 


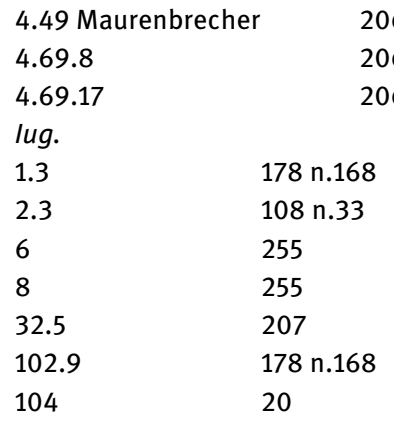

\section{Pseudus Sallustius}

rep.

2.1.2

178 n.168

Senatus consultum de Cn Pisone patre

$\begin{array}{ll}\text { I. } 28 & 262 \\ \text { II. } 45-49 & 262\end{array}$

\section{Seneca, Lucius Annaeus (rhetor)}

contr.

1 praef.

1 praef. 1

1 praef. $1-4$

1 praef. $1-12$

1 praef. 2-5

1 praef. 6

1 praef. 6-7

praef. $7-10$

1 praef. 7

praef. 8-10

1 praef. 8-11

1 praef. 8

1 praef. 9

1 praef. $10-11$

1 praef. 11

206

206

206

n. $165 ; 178$ n.166

110; 117 n. $55 ; 153$

n.158; 185 n.206

193

n. 55

153 n.44; 157 n. 65

158 n.69

93; 127 n. $19 ; 148$ n. $64 ; 175$ n. $156 ; 178$

n. $45 ; 171$ n. $137 ; 176$

n. 20; 154 n. 49; 154
1 praef. 12

1 praef. $13-24$

1 praef. 18

1 praef. 19

1 praef. 20

1.1.3

1.1.5

1.1.17

1.1.23

1.2

1.2.3

1.2.23

1.3.10

1.4.1

1.4.11

1.5

1.6

1.6.8

1.6.10-12

1.6.12

1.7

1.7 .4

1.7.13

1.8.12

1.8.16

2 praef.

2 praef. 1

n. $50 ; 159$ n.70; 159

n.71; 160 n.78;

162n.88; 167 n.117;

169 n.128; 170 n.133;

180 n.175; 182 n.190;

318 n. $12 ; 348$ n.69

135; 146 n. $15 ; 164$

n.103; 176 n. $157 ; 178$

n.166

155 n. $53 ; 185$ n.201

180 n.177

180 n.180; 180 n.181;

182 n.184

180 n.175

178 n.167

178 n.167

178 n.167

153 n. 45

182 n. 185

169 n.127

182 n.185

265

207

169 n.127

182 n.185

137 n.64

$137 \mathrm{n} .64$

$137 \mathrm{n} .64$

44 n. 45 ; 81 n. 30 ;

$141 \mathrm{n} .77$

286

285

181 n.179

152 n. 34; 152 n.37;

178 n.167; 179 n.173

309 n. 53

165 n.107; 180 n.181;

304 n.39; 307 n.50;

309 n. 54

2 praef. $2 \quad 113$ n.44; 304 n.39;

311 n. 60

2 praef. $3 \quad 135 ; 152$ n. $34 ; 188$

n.218; 304 n.39

2 praef. 3-4

171 n.138; 172 n.144;

180 n. $181 ; 182$ n.188 


\begin{tabular}{|c|c|c|c|}
\hline \multirow[t]{2}{*}{2 praef. 5} & 135 n.56; 152 n.34; & 4 praef. 2-6 & 156 n.58 \\
\hline & 153 n.45; & 4 praef. 3-6 & 167 n.118 \\
\hline 2.1 & 113 n.43 & 4 praef. 3 & 167 n.118 \\
\hline 2.1 .1 & 178 n.167; 180 n.178 & 4 praef. 5 & 168 n.121 \\
\hline \multirow[t]{2}{*}{2.1 .7} & 133 n.45; 178 n.167; & 4 praef. $6-7$ & 81 n.30 \\
\hline & 180 n.178 & 4 praef. 7 & 81 n.31; 156 n.58; 284 \\
\hline 2.1.10-11 & $112 ; 117$ n.55 & 4.3 & $141 \mathrm{n} .77$ \\
\hline 2.1.11 & 116 & 4.8 & 169 n.129 \\
\hline 2.1 .12 & 113 n.45; 116 & 5.1 & 169 n.129 \\
\hline 2.1 .25 & 113 n.43 & 5.6 & 182 n.185 \\
\hline 2.1.37 & 187 n.212 & 6.4 & 82 n.37 \\
\hline 2.2 & 137 & 7 praef. & 180 n.182 \\
\hline 2.2 .2 & 137 n.65 & 7 praef. 1 & 180 n.181 \\
\hline 2.2 .5 & 137 n.65 & 7 praef. 2 & 187 n.211; 311 n.60 \\
\hline \multirow[t]{2}{*}{$2.2 .8-12$} & 182 n.186; 187 n.212; & 7 praef. $4-6$ & 123 n.2 \\
\hline & 189 n.221; 190 n.225 & 7 praef. 5 & 157 n.64; 188 n.217 \\
\hline 2.2 .8 & 137 & 7 praef. $6-7$ & $135 ; 310$ n.59 \\
\hline 2.2 .12 & 266 n.24 & 7 praef. 6 & 310 n.59 \\
\hline 2.3 .13 & 135 & 7.1.4 & 44 n. $45 ; 81$ n.30 \\
\hline 2.3 .15 & 185 n.211 & 7.1.24 & 44 n.45; 81 n.30 \\
\hline 2.4 .4 & 285 & 7.1 .27 & 249 n.83 \\
\hline $2.4 .6-8$ & 187 n.212 & 7.2 & 169 n.129; 170 n.133 \\
\hline 2.4 .8 & 156 n.58; 182 n.187 & 7.2 .5 & 44 n.45; 81 n.30; 223 \\
\hline $2.4 .12-13$ & 46 n.51; 156 n.58; 322 & & n.96 \\
\hline 2.4 .12 & 156 n.58 & 7.2 .8 & $135 ; 159$ n.72 \\
\hline \multirow[t]{3}{*}{2.4 .13} & 156 n.58; 168 n.121; & $7.3 .8-9$ & 187 n.212 \\
\hline & 171 n.139; 187 n.212; & 7.4 & $141 \mathrm{n} .77$ \\
\hline & 284 & 7.4 .6 & 151 n.33; 170 n.133 \\
\hline 2.5 .7 & 176 n.158 & $7.4 .6-8$ & 135 \\
\hline 2.5 .12 & 285 & 7.5 .11 & 169 n.127; 249 n.83 \\
\hline 2.5 .17 & 136 n.61 & 7.6.11 & 169 n.127 \\
\hline 2.5 .19 & 169 n.127 & 7.6.18 & 178 n.167 \\
\hline 2.5 .20 & 156 n.58; 284 & 7.5.12 & 135 \\
\hline \multirow[t]{2}{*}{2.6 .2} & 112 n.46; 113 n.46; & 7.6.22 & 135 \\
\hline & 176 n.158 & 7.8 .3 & 44 n.45; 81 n.30 \\
\hline 2.7 & 113 & 9 praef. & 153 n.42; 179 n.173; \\
\hline 2.7 .1 & 176 n.158 & & 180 n.182 \\
\hline 3 praef. & 179 n.173 & 9 praef. 1 & 180 n.181 \\
\hline 3 paref. 1 & 180 n.181 & 9.1 .13 & 156 n.57; 163 n.95; \\
\hline 3 praef. 3 & 153 n.42; 171 n.139 & & 164 n.101 \\
\hline \multirow[t]{2}{*}{3 paref. 8} & 122 n.73; 156 n.57; & $9.1 .13-14$ & 122 n.73; 156 n.57; \\
\hline & 161 n.84 185 n.205 & & 157 n.64; 161 n. 84 \\
\hline 3 praef. 15 & 151 n.33; 170 n.133 & & 176 n.158; 187 n.212; \\
\hline 3 exc. 9 & $95 \mathrm{n} .24$ & & 189 n.222 \\
\hline 4 praef. & 182 n.185 & 9.1 .14 & 156 n.57; 189 n.222 \\
\hline 4 praef. 1 & 180 n.181; 183 n.193 & 9.2 .9 & 95 \\
\hline 4 praef. 2 & 156 n.58; 180 n.181 & 9.2.19 & 285 \\
\hline
\end{tabular}




\begin{tabular}{|c|c|c|c|}
\hline $9.2 .23-24$ & 187 n.212 & 10.5 .22 & 153 n.42; 156 n.57; \\
\hline 9.2 .24 & 309 n.54 & & $161 \mathrm{n} .83$ \\
\hline \multirow[t]{2}{*}{9.2 .26} & 156 n.57; 161 n.85; & 10.5 .21 & 89 \\
\hline & 189 n.223; 189 n.224 & 10.5 .24 & 44 n. $45 ; 81$ n.30 \\
\hline 9.3.12-13 & 187 n.212 & 10.6 & 141 n.77 \\
\hline 9.3 .13 & 81 n. 30 & 10 exc. 3 & 95 n.24 \\
\hline 9.3 .14 & 44 n. $45 ; 156$ n.58 & exc. 4 praef. 6-11 & 44 n.45; \\
\hline 9.4 & 285 & 11 & 151 n.33 \\
\hline $9.4 .4-6$ & 285 & suas. & \\
\hline 9.4 .4 & 285 & 1.3 & 108 n.33; 286 n.39 \\
\hline 9.4 .16 & 44 n.45; 81 n.30 & 1.5 & $100 ; 163$ n.91; 166 \\
\hline $9.4 .17-21$ & 168 n.120; 187 n.212 & & n.94; 163 n.97; 187 \\
\hline 9.4 .19 & 249 n.83 & & n.212; 188 n.214; \\
\hline $9.5 .15-17$ & 187 n.212 & & 196; 268 n.30; 270; \\
\hline 9.5.17 & 190 n. $225 ; 222$ & & 286 \\
\hline 9.6 .8 & 44 n. $45 ; 81$ n.30 & $1.5-7$ & 268 \\
\hline 9.6.11 & 81 n.30; 301 n.29 & $1.6-7$ & 268 \\
\hline 9.6 .13 & 81 n.30 & 1.7 & 10 n. $8 ; 123$ n. $19 ; 154$ \\
\hline 9.6.16 & 81 n. 30 & & n.52; 156 n.57; 156 \\
\hline 9 exc. 2 & $95 \mathrm{n} .24$ & & n. $58 ; 187$ n. $212 ; 268$ \\
\hline 10 & 156 n.58 & 1.9 & 180 n.178 \\
\hline 10 praef. & 185 n.201 & 1.12 & 156 n.58 \\
\hline 10 praef. 1 & $\begin{array}{l}152 \text { n.34; } 179 \text { n.173; } \\
180 \text { n.181 }\end{array}$ & 1.15 & $\begin{array}{l}155 \text { n.55; } 156 \text { n.57; } \\
157 \text { n.62; } 189 \text { n.219; }\end{array}$ \\
\hline 10 praef. 2 & 156 n.57 & & 268 n.28 \\
\hline 10 praef. 3 & 153 n. $42 ; 171$ n.139 & $1.15 .16-23$ & 266 \\
\hline 10 praef. $4-8$ & 156 n.58 & 2.2 & 181 n.179 \\
\hline \multirow[t]{2}{*}{10 praef. 5} & 156 n.57; 161 n.83; & $2.5-7$ & 187 n.212 \\
\hline & 167 n.114 & 2.11 & 164 n.102; 166 n.96; \\
\hline 10 praef. $5-7$ & 171 n.139 & & 196 \\
\hline 10 praef. 6 & 151 n.33; 170 n.133 & 2.12 & 165 n.107; 171 n.142 \\
\hline 10 praef. $6-7$ & 153 n. $43 ; 185$ n.206 & $2.12-13$ & 187 n.212 \\
\hline 10 praef. 7 & 144 n.4; 153 n.42 & 2.14 & 81 n. $30 ; 161$ n. $82 ; 44$ \\
\hline 10 praef. $7-8$ & 156 n.57 & & n. 45 \\
\hline 10 praef. 8 & 128 n.22; 161 n.83 & 2.15 & 182 n.185; 187 n.212; \\
\hline 10 praef. 12 & 302 n.32 & & $265 ; 266$ \\
\hline 10 praef. 13 & 185 n.200 & 2.17 & 155 n.53; 187 n.212 \\
\hline 10 praef. $14-16$ & 167 n.113 & $2.19-20$ & 187 n.212 \\
\hline 10 praef. 14 & 156 n.58; 168 n.120 & 2.19 & 151 n.33; 158 n.69; \\
\hline 10 praef. 16 & 159 n.71; 169 n.130 & & 170 n.133 \\
\hline 10.1 .8 & $95 \mathrm{n} .24$ & 2.20 & 156 n.58 \\
\hline 10.2 .19 & 156 n.58 & 2.22 & 153 n. $42 ; 161$ n.82 \\
\hline 10.3 .3 & 95 n.24 & $3.6-7$ & $44 \mathrm{n} .45$ \\
\hline 10.4.18 & 176 n.158 & 3.6 & 156 n.58; 249 n.83 \\
\hline 10.4 .25 & 169 n.127 & 3.7 & 44 n.45; 312 n.3; 81 \\
\hline 10.5 .20 & 158 n.69 & & n.31 \\
\hline $10.5 .21-22$ & 187 n.212 & 4.4 & 187 n.212 \\
\hline
\end{tabular}




\begin{tabular}{|c|c|c|c|c|}
\hline 4.5 & 161 n.4.5 & 6.22 & \multicolumn{2}{|c|}{156 n.57; 160 n.75; } \\
\hline \multirow[t]{2}{*}{5.8} & 152 n.34; 152 n.35; & & \multicolumn{2}{|c|}{170 n.134; 184 n.196; } \\
\hline & 179 n.171; 179 n.173 & & \multicolumn{2}{|c|}{189 n.224 } \\
\hline \multirow[t]{3}{*}{6} & $135 ; 148$ n. $21 ; 148$ & 6.23 & \multicolumn{2}{|c|}{156 n.57; 157 n.64; } \\
\hline & n.22; 158; 170 n.133; & & \multicolumn{2}{|c|}{160 n.76; 160 n.77; } \\
\hline & 196 & & \multicolumn{2}{|c|}{161 n.87; 211 n.43 } \\
\hline $6.1-2$ & $44 \mathrm{n} .45$ & $6.24-25$ & \multicolumn{2}{|c|}{161 n. 81} \\
\hline 6.1 & 81 n.30 & 6.24 & \multicolumn{2}{|c|}{156 n. $57 ; 161$ n. 80} \\
\hline \multirow[t]{2}{*}{6.3} & 163 n.98; 181 n.179; & $6.25-26$ & \multicolumn{2}{|c|}{155 n.56 } \\
\hline & $196 ; 285$ & 6.25 & \multicolumn{2}{|c|}{130 n.28; 189 n.220 } \\
\hline $6.5-6$ & 180 n.174 & 6.26 & \multicolumn{2}{|c|}{156 n. $57 ; 157$ n.62 } \\
\hline 6.6 & 82 n.37 & 6.27 & \multicolumn{2}{|c|}{156 n.58; 183 n.194; } \\
\hline 6.8 & 180 n.178 & & \multicolumn{2}{|c|}{189 n.220; 189 n.221 } \\
\hline 6.11 .14 & 95 n.24 & 7 & \multicolumn{2}{|c|}{148 n.21; 148 n.22; } \\
\hline 6.12 & 151 n.33 & & \multicolumn{2}{|c|}{170 n.133 } \\
\hline $6.14-16$ & 135 & $7.1-3$ & \multicolumn{2}{|c|}{44 n. $45 ; 287$ n.45 } \\
\hline $6.14-15$ & 170 n.133; 183 n.194 & 7.1 & \multicolumn{2}{|c|}{81 n.30 } \\
\hline $6.14-21$ & 187 n.212 & 7.3 & \multicolumn{2}{|c|}{287} \\
\hline $6.14-25$ & 19 n.42 & 7.4 & \multicolumn{2}{|l|}{287} \\
\hline $6.14-27$ & 151 n.33; 170 n.133 & 7.5 & \multicolumn{2}{|c|}{95 n.24 } \\
\hline 6.14 & 95 n.24; 139 n.70; & 7.10 & $151 r$ & \\
\hline & 158 n.68; 159; 159 & $7.13-14$ & $151 r$ & 3; 170 n.133; \\
\hline & n.71; 162 n.88 & & 187 & 12 \\
\hline 6.15 & 139; 156 n.57; 170 & 7.14 & $151 r$ & \\
\hline & n.73; 170 n.74; 161 & hist, frg. & 67) & \\
\hline & n.80 & cr. $1 \mathrm{pz}$ & & 80 n.22 \\
\hline 6.16 & $92 ; 98 ; 152$ n.34; 152 & cr. 1 pz. I & & 78 n.11 \\
\hline & n.36; 152 n. 38; 179 & cr. 1 pz. I & & 80 n.19; 262; \\
\hline & n.171; 181 n.181; 302 & cr. 2 pz. I & & 43 n. $41 ; 47$ \\
\hline & n.34 & & & $\mathrm{n} .56$ \\
\hline $6.16-17$ & 184 n.196 & cr. 2 pz. I & & 43; 76 n.4; \\
\hline $6.16-24$ & $123 ; 179$ n.173 & & & 79 n.18 \\
\hline $6.16-25$ & 102 n.3 & cr. 2 pz. I & & 48 n.57 \\
\hline $6.17-21$ & $139 ; 156$ n. 57 & cr. 2 pz. I & col. 1 & $2-5 \quad 43 n .41$ \\
\hline 6.17 & 95 n.24; 189 n.224 & cr. 2 pz. I & 11.4 & 264 \\
\hline $6.18-19$ & 160 n.74 & cr. 2 pz. I & & $36 ; 84$ n.56 \\
\hline 6.18 & 156 n.57; 160 n.76; & cr. 2 pz. I & & 84 n.56 \\
\hline & 160 n.77 & cr. 2 pz. I & I. II II. & 36 \\
\hline 6.19 & 95 n.24; 156 n.57 & cr. 2 pz. I & & 47 n.56 \\
\hline $6.20-21$ & 156 n.57; 161 n.87 & cr. 2 pz. I & +10 & 81 n.33 \\
\hline $6.21-22$ & 161 n.86 & cr. 2 pz. I & $1-10$ & 43 n.43 \\
\hline $6.21-27$ & 187 n.212 & cr. 2 pz. I & $9-10$ & 79 n.12 \\
\hline 6.21 & 122 n.73; 137; 155 & cr. 2 pz. I & & 69 \\
\hline & n.21; 156 n.57; 176 & cr. 3 pz. I & & 48 n.57 \\
\hline & n.159; 189 n.224; 211 & cr. 3 pz. I & & 36 n.25; 78 \\
\hline & & & & n.11 \\
\hline & & cr. 3 pz. I & & 47 n.56 \\
\hline
\end{tabular}




\begin{tabular}{|c|c|c|c|c|}
\hline \multirow{3}{*}{$\begin{array}{l}\text { cr. } 3 \text { pz. I sov. } 3 \text {, l. } 8 \\
\text { cr. } 3 \text { pz. I sov. } 4 \\
\text { cr. } 3 \text { pz. I sov. } 7 \text { (?) }\end{array}$} & $44 ; 79$ n.12 & \multicolumn{2}{|c|}{ cr. 6 pz. II sov. $1+$ sov. 2} & 47 n. $56 ; 48$ \\
\hline & 47 n.56 & & \multicolumn{2}{|c|}{ n. 57} \\
\hline & 47 n. $56 ; 48$ & \multicolumn{2}{|c|}{ cr. 6 pz. II sov. $1+$ sov. 2 Il. $4-5$} & 79 n.14 \\
\hline & $\mathrm{n} .57$ & \multicolumn{2}{|c|}{ cr. 6 pz. II sov. $1 \mathrm{l} .4$} & $76 \mathrm{n} .4$ \\
\hline \multirow[t]{2}{*}{ cr. 3 pz. I sov. 8} & 47 n.56; 48 & cr. 7 & \multicolumn{2}{|l|}{38 n.32 } \\
\hline & n.57 & cr. 7 pz. II & \multicolumn{2}{|l|}{34 n.13 } \\
\hline cr. 3 pz. I sov. 8 , col. I l. 2 & 83 n.47 & cr. 8 pz. II & \multicolumn{2}{|l|}{34 n.13 } \\
\hline \multicolumn{2}{|l|}{ cr. 3 pz. I sov. 8, col. I ll. 9-10 } & cr. 9 pz. III & \multicolumn{2}{|l|}{34 n.13; 37} \\
\hline \multicolumn{2}{|l|}{ cr. 3 pz. I sov. 8 col. II II. 4-17 } & & & \\
\hline cr. 3 pz. I sov. 8 Il. 7 & 264 & \multicolumn{3}{|c|}{ Seneca, Lucius Annaeus } \\
\hline cr. 3 pz. I sov. 8 II.10 & 264 & \multicolumn{3}{|l|}{ apocol. } \\
\hline cr. 4 pz. I sov. 2 & 47 n.56 & 1 & 92 & \\
\hline cr. 4 pz. I sov. 2 Il. 5-7 & $78 \mathrm{n} .10$ & 5 & 92 & \\
\hline cr. 4 pz. I sov. 4 & 47 n.56 & benef. & & \\
\hline cr. 4 pz. I strato 1 & $48 \mathrm{n} .57$ & 1.10 .1 & 176 n.161 & \\
\hline cr. 4 pz. I strato 1 l. 4-6 & 79 n.13 & 1.13 .3 & 286 n.41 & \\
\hline cr. 5 & 56 & 2.16 .1 & 286 n.41 & \\
\hline cr. 5,1 & 57 & 2.20 & 90 & \\
\hline cr. 5 pz. I & 34 n.13; 36 & 2.20 .2 & 170 n.135 & \\
\hline & n.27 & 2.21 .5 & 284 & \\
\hline cr. 5 pz. I sov. 1 & $44 ; 46 ; 46$ & 3.7.7 & 133 n.45 & \\
\hline & n.52; 47; 47 & $3.27 .1-4$ & 168 n.121 & \\
\hline & n.56; 48 n.57 & 5.15 .5 & 79 n.15 & \\
\hline cr. 5 pz. I sov. 1, col. I & $44 \mathrm{n} .48$ & $5.16 .2-5$ & 283 & \\
\hline cr. 5 pz. I sov. 1 , col. I l. 1 & 44 & 6.32 .4 & 348 n.70 & \\
\hline cr. 5 pz. I sov. 1 , col. I l. 1-1 & $83 n .48$ & $6.34 .1-2$ & 99 & \\
\hline cr. 5 pz. I secondo intercolu & mnio l. 966 & $7.2 .5-6$ & $271 ; 271$ n.3 & \\
\hline cr. 5 pz. I sov. 1 , col. II & 46 & clem. & & \\
\hline cr. 5 pz. I sov. 1 l. 1 & 79 n.11 & 1.1.6 & $275 ; 275$ n.4 & \\
\hline cr. 5 pz. I sov. $1 \mathrm{l} .3$ & $76 n .4$ & 1.1.7 & 117 n.61 & \\
\hline cr. 5 pz. I sov. 1 l. 4 & 79 n.11 & 1.3 .5 & 114 n.49 & \\
\hline cr. 5 pz. I sov. 1 l. 7 & 79 n.11 & 1.5.1 & 45 n.49 & \\
\hline cr. 5 pz. I sov. 5 l. 10 & 78 n.11 & 1.9 & 333 & \\
\hline cr. 5 pz. II & $34 \mathrm{n} .13$ & 1.9 .3 & 271 & \\
\hline cr. 5 pz. II sov. 1 & 47 n. $56 ; 48$ & dial. & & \\
\hline & n. 57 & 1.3 .10 & 117 n.61 & \\
\hline cr. 5 pz. II sov. 1 , Il. 1-8 & & 1.4 .6 & 117 n.61 & \\
\hline cr. 5 pz. II sov. 1 , Il. 7-8 & $76 n .4$ & 2.1 .3 & 284 & \\
\hline cr. 5 pz. II sov. 1, l. 7 & 47 & 2.5 .4 & 108 n.33 & \\
\hline cr. 5 pz. II sov. 1 , I. 8 & $47 ; 66$ & $2.18 .1-3$ & 284 & \\
\hline cr. 5 pz. II sov. 2 & 48 n. 57 & 4.2.33.1-4 & 284 n.34 & \\
\hline cr. 5 pz. II sov. 3 & $48 \mathrm{n} .57$ & 4.3.3 & 99 & \\
\hline cr. 5 pz. II sov. 3 II. 3-6 & 78 n.11 & 4.3.18.1 & 282 n.26 & \\
\hline cr.6 & $56 ; 57$ & 4.3.18.4 & 284 & \\
\hline cr. 6,2 & 57 & 4.3.22.1 & 145 n.12 & \\
\hline cr. 6,3 & 57 & 4.3.23.7-8 & 161 n.121 & \\
\hline cr. 6 pz. II & 42 & 6.1.2-4 & 128 n.26; 14 & n.24 \\
\hline
\end{tabular}




\begin{tabular}{|c|c|c|c|}
\hline $6.1 .3-4$ & 179 n.172 & 100.12 & $294 \mathrm{n} .3$ \\
\hline 6.1 .3 & 153 n. 42 & $104.29-32$ & 283 \\
\hline 6.12 .2 & 95 & 108 & $295 ; 295$ n.5 \\
\hline 6.15 .3 & $273 ; 273$ n. $44 ; 275$ & 108.22 & $92 ; 165$ n.105; 295 \\
\hline 6.23 .3 & 108 n.33 & & n.6; 300 n.23 \\
\hline 11.8 .2 & $151 \mathrm{n} .33$ & 108.31 & 135 n. 54 \\
\hline 11.14.1 & 272 & 109.16 & 135 n.222; 189 n.222 \\
\hline 11.14.2-11.16.3 & 271 & 110.7 & 309 n.54 \\
\hline $11.16 .1-2$ & 272 n. $40 ; 275$ & 114 & $295 \mathrm{n} .4$ \\
\hline 11.16 .3 & 272 n.42 & 114.1 & 295 n.4 \\
\hline $12.2 .4-5$ & 316 n.3 & 114.7 & 309 n.54 \\
\hline \multirow[t]{2}{*}{$12.17 .3-4$} & 148 n.19; 153 n.44; & 114.8 & 117 n.61 \\
\hline & 165 n.105 & $114.15-16$ & 296 n. 8 \\
\hline 12.17.3 & 153 n.44; 166 n.108 & 114.16 & 308 n.51 \\
\hline $12.18 .1-3$ & 170 n.131 & 114.17-19 & 189 n.222; 204 \\
\hline 12.19 .2 & 169 n.126 & $114.17-21$ & 189 n.222 \\
\hline epist. & & 122.15 & 266 \\
\hline $14.13-13$ & 283 & nat. & \\
\hline 14.13 & 170 n.135 & 1.1 .3 & 273 n.43 \\
\hline 20.5 & 189 n.222 & 3 praef. 5 & 145 n.12 \\
\hline 21.4 & 141 n.76 & 3 praef. 7 & 92 \\
\hline $38.1-2$ & 313 n.64 & 4a praef. 21 & 92 \\
\hline 40 & 295 n.4 & 4b.3.1 & 92 \\
\hline 40.10 & 81 n.30 & $6.23 .2-3$ & 270 n.36 \\
\hline 60.4 & 189 n.222 & $6.23 .2-4$ & 163 n.94 \\
\hline 83.13 & 145 n.12 & 7.16 .1 & 92 \\
\hline 83.25 & $271 ; 271$ n.39 & & \\
\hline 91.5 & 117 n.61 & \multicolumn{2}{|c|}{ Pseudus Seneca } \\
\hline 91.17 & 286 n.41 & epist. Paul. 2 & 89 \\
\hline $94.62-63$ & $283 ; 286$ & & \\
\hline $94.65-67$ & 283 & \multicolumn{2}{|c|}{ Sidonius, Caius Sollius Apollinaris } \\
\hline 95.70 & 283 & carm. & \\
\hline 100 & 294 & $9.230-238$ & 88 \\
\hline $100.1-2$ & 298 & 23.162 & 88 \\
\hline 100.1 & 294 n.3; 299 n.21 & & \\
\hline 100.2 & 300 n.22; 300 n.23 & Strabo & \\
\hline 100.4 & 310 n.59 & 3.2 .1 & 166 n.110 \\
\hline $100.3-4$ & 298 & 3.2 .15 & 166 n.110 \\
\hline 100.5 & $298 ; 306$ n.42 & & \\
\hline 100.6 & 307 n.45 & \multicolumn{2}{|c|}{ Suetonius Tranquillus, $\mathrm{C}$. } \\
\hline 100.7 & 308 n.51; 311 n.62 & \multicolumn{2}{|l|}{ Aug. } \\
\hline 100.8 & 306 n.42; 306 n.43; & 29.3 & $131 ; 132$ n.41 \\
\hline & 307 n.47 & 32.3 & $131 ; 131$ n. $35 ; 132$ \\
\hline 100.9 & 302 n.35 & & n.39 \\
\hline 100.10 & 306 n.44 & 50 & 138 \\
\hline $100.11-12$ & 298 n.16 & 65.1 & 84 n.55 \\
\hline 100.11 & 306 n.42; 307 n.46 & 101.2 & 84 n.49 \\
\hline
\end{tabular}




\begin{tabular}{|c|c|c|c|}
\hline Cal. & & 7.2 & $125 \mathrm{n} .10$ \\
\hline 1.2 & 141 n.75 & 9.1 & 126 n.17 \\
\hline $3.1-2$ & $265 ; 265$ n. 22 & 10 & 126 n.17 \\
\hline 4.1 & $47 ; 84$ n.53 & 11.5 & $126 \mathrm{n} .17$ \\
\hline 5 & 141 n.76 & 13 & 89 \\
\hline 6.3 & 276 & 15.2 & 84 n. 53 \\
\hline 8.2 & 124 n.7 & 18.1 & 78 n.10 \\
\hline 11 & 124 n. 8 & 21.1 & 125 n.10 \\
\hline $12.2-3$ & 138 n.66 & 21.3 & 78 n.10 \\
\hline \multirow[t]{2}{*}{12.3} & 138; 138 n.68; $140 ;$ & 21.5 & 78 n.10 \\
\hline & 260 n.5 & 22 & 124 n.8; 126 n.17 \\
\hline $13-16$ & 128 n.26; 129 n.27 & 24.1 & $124 \mathrm{n} .8$ \\
\hline 14.3 & 140 n.72; 141 n.76 & 25.1 & 124 n. $8 ; 250$ \\
\hline 15.1 & 139 & 25.3 & 126 n.18 \\
\hline $15.2-3$ & 140 & 26.2 & 84 n. $50 ; 124$ n. $8 ; 126$ \\
\hline 15.2 & 140 & & n.18 \\
\hline 16.1 & 125 & 27.1 & 82 n. $34 ; 82$ n. $35 ; 126$ \\
\hline 16.2 & $132 ; 132$ n.41 & & n.14 \\
\hline 61.6 & 125 & 28 & $124 \mathrm{n} .8$ \\
\hline Claud. & & 29 & $124 \mathrm{n} .8$ \\
\hline 11.2 & 139 n.71 & 29.1 & 81 n.32 \\
\hline 11.3 & 272 n.41; 272 n.41 & 32.1 & $124 \mathrm{n} .8$ \\
\hline 15.1 & 132 n.39; 132 n.41 & 32.2 & $124 \mathrm{n} .8$ \\
\hline 21.2 & 135 & 34.1 & 124 n. 8 \\
\hline 23.1 & 132 n.39 & 34.2 & 124 n. $8 ; 126$ n.18 \\
\hline 41 & 149 n.23 & 35.1 & 136 \\
\hline $41-42$ & 128 & 35.2 & 126 n.17; 136 \\
\hline 41.1 & 24 & 37.1 & 127 \\
\hline 41.2 & 348 n.70; 348 n.70 & 37.3 & 126 n.17 \\
\hline 41.3 & 151 n.33 & 38 & 125 \\
\hline Dom. & & 39.1 & 276 \\
\hline $8.1-3$ & 132 n.41 & 41 & $130 ; 131$ \\
\hline 20 & 124 n. 8 & 42.2 & 137 n.63 \\
\hline Gal. & & $42-72$ & $125 \mathrm{n} .13$ \\
\hline 4.1 & 124 n. 8 & 43.2 & 125 n.10 \\
\hline 14.3 & 132 n.39; 134 n.51 & 45 & 125 n.10 \\
\hline 15.1 & 134 n.50 & 47 & 124 n.8; 131 \\
\hline lul. & & 49 & 79 n.17; 80 n.23; 125 \\
\hline 56.1 & 79 n.15 & & n.13 \\
\hline 69.1 & 79 n.15 & 49.2 & 125 n.13; 141; 141 \\
\hline Tib. & & & n.75 \\
\hline 1.1 & 126 n.17 & 50 & $124 \mathrm{n} .8$ \\
\hline 1.2 & 125 & 51.1 & $131 ; 137$ n.63 \\
\hline 3.1 & 84 n.54 & 51.2 & $126 ; 126$ n.14 \\
\hline 3.2 & 126 n.17 & 52 & 80 n.25 \\
\hline $4-41$ & 125 & 52.2 & 126 n.18 \\
\hline 5.1 & 126 n.17 & 52.3 & 125 n.10 \\
\hline
\end{tabular}




\section{3}

53.1

53.2

54.2

57.2

58

58.1

59

59.1

59.2

59.3.8

61

61.2

61.3

61.4

61.5

61.6

62

62.1

62.3

63

65.1

66

66.2

67.1

67.3-4

72.1

73

73.2

75.1

75.2

75.2

Tit.

6.2

rhet.

1

$$
6
$$

30.4

Vit.

2.4
126

82 n. $41 ; 124$ n. 8

124 n. 8

139

$124 \mathrm{n} .8$

$126 \mathrm{n} .18$

124 n. 8

125

125 n. 11

124 n. 8

139

153 n. 42

127

24; 83 n. $46 ; 128$

82 n. $42 ; 127$

$124 \mathrm{n} .8$

239; 241

127

$83 \mathrm{n} .43$

$124 \mathrm{n} .8$

126 n. 18

124 n. 8

140

84 n. 52

$124 \mathrm{n} .8$

124 n. 8

126 n. 18

126 n. $17 ; 143$ n. $2 ; 144$

n.4; 144 n. $6 ; 192$

64 n.60; 103 n.9; 123;

195; 250; 260 n.5;

316 n. $3 ; 343 ; 355$

141

141

142

209 n.31

123 n.2

$144 \mathrm{n} .4$

123 n.2

141 n.76
Tacitus, P. Cornelius

Agr.

21.2

30.5

223 n.95

223 n. 5

ann.

1-6 7, 243; 243 n.41; 245;

246 n. 62

1.1

91; 159 n.71; 193

n. 229

1.1.2

$16 ; 128$ n. $21 ; 246$

183 n.192

84 n. $53 ; 261$ n. 9

22

248

250 n. 89

84 n. 55

126 n.18

253

252

280 n.13

1.9.4

$\begin{array}{ll}1.10 .8-1.15 .2 & 252 \\ 1.12 .4 & 251\end{array}$

1.13.2 14

1.13.3-6 $81 \mathrm{n.28}$

1.13 .6

1.13 .7

$82 ; 126$ n.14

82 n. 35

80 n.23

261 n.10

240

252

266

241; 245

125 n.11

244; 252

$144 \mathrm{n} .4$

126 n. $14 ; 244$ n.47

244

244

244

141 n.75

246

268

268 n.29

253

253

126 n.18

250 


\begin{tabular}{|c|c|c|c|}
\hline 2.32 & 253 & $3.20-21$ & 253 \\
\hline 2.32 .3 & 126 n.14 & $3.22-23$ & 253 \\
\hline 2.39 .2 & 248 & 3.23 .2 & $82 \mathrm{n} .40$ \\
\hline 2.40 .2 & 250 & 3.25 .1 & 253 \\
\hline 2.41 .2 & 80 n. $20 ; 263$ n.14 & 3.26 .3 & 252 \\
\hline 2.43 & 263 & 3.27 & 98 n.27 \\
\hline $2.43 .2-4$ & 262 & 3.29 .1 & 253 \\
\hline $2.43 .5-6$ & 246 n.62; 263 & 3.29 .4 & 209 n.31 \\
\hline 2.43 .6 & 264 & 3.30 .1 & 131 n.36; 132 n.41 \\
\hline 2.47 & 253 & $3.30 .2-3$ & 248 \\
\hline 2.52 & 253 & 3.31 & 253 \\
\hline 2.53 .1 & 126 n.18 & 3.31 .1 & 126 n.18 \\
\hline 2.53 .2 & 269 & 3.32 & 253 \\
\hline 2.53 .3 & 269 & $3.33-34$ & 253 \\
\hline 2.55 .1 & 270 n.33 & 3.34 & 176 n.161 \\
\hline $2.56 .2-3$ & 141 n.75 & 3.35 & 253 \\
\hline 2.58 & 141 n.75 & 3.38 .1 & 253 \\
\hline $2.62-63$ & 253 & $3.38 .2-39$ & 253 \\
\hline 2.63 .4 & 247 n.73 & 3.38 .2 & 253 \\
\hline 2.64 .1 & 263 n.15 & $3.40-47$ & 43 n.39; 79n.17; 253 \\
\hline 2.68 & 141 n.75 & 3.4 & 79 \\
\hline $2.69 .2-3$ & 253 & 3.48 .1 & 253 \\
\hline 2.69 .3 & 82 n.38 & $3.49-50$ & $244 ; 253$ \\
\hline 2.70 .2 & 244 & 3.49 & 80 \\
\hline 2.71 & 253 & 3.52 & 253 \\
\hline $2.73 .1-3$ & 267 n.27 & 3.55 & 176 n.161 \\
\hline 2.74 & 80 n.24 & $3.56 .3-4$ & 253 \\
\hline 2.83 & $253 ; 264$ & 3.57 & 253 \\
\hline 2.83 .3 & 265 n.23 & 3.57 .2 & $81 \mathrm{n} .28$ \\
\hline $2.85 .1-2$ & 136 & $3.58-59$ & 253 \\
\hline $2.85 .1-2$ & 136 & 3.59 .2 & 253 \\
\hline 2.85 .4 & 126 n. $14 ; 253$ & $3.60-63$ & 253 \\
\hline 2.86 & 253 & 3.64 & 253 \\
\hline 2.88 .3 & 241 n.31 & $3.66-68$ & 253 \\
\hline $3.1-9$ & 80 n.25 & 3.68 & 80 n.23 \\
\hline 3.3.2 & 243 n. 40 & 3.69 & 253 \\
\hline 3.7 & 80 n.24 & 3.69 .5 & 78 n.10 \\
\hline 3.7 .2 & 82 n.38 & 3.70 .1 & 253 \\
\hline 3.11 .1 & $263 ; 263$ n.15 & 3.71 & 253 \\
\hline $3.12-19$ & 253 & 3.71 .1 & 253 \\
\hline 3.12 .4 & 82 n.38 & 3.72 .1 & 253 \\
\hline 3.13 .2 & 82 n.38 & 3.72 .2 & 253 \\
\hline 3.14 .1 & 82 n.38 & 3.72 .4 & 253 \\
\hline 3.16 .1 & 244 & $3.73-74$ & 253 \\
\hline 3.17 .4 & 253 & 3.75 .1 & 251 \\
\hline 3.18 .2 & 253 & 3.76 & 251 \\
\hline 3.19 .3 & 82 n.39 & 4.2.1 & 127 \\
\hline
\end{tabular}




\begin{tabular}{|c|c|c|c|}
\hline 4.6.1 & $275 ; 276$ n.50 & 6.51 .1 & 84 n.54 \\
\hline 4.6 .4 & 78 n.10; 247 n.68 & 6.51 .3 & $253 ; 276$ \\
\hline 4.7.1 & 276 & $11-16$ & 243 \\
\hline $4.8-11$ & 264 n.11 & 11.18 .2 & 228 n.118 \\
\hline 4.9 .2 & 264 & 11.21 & 14 \\
\hline $4.10-11$ & 83 n.43 & 11.24 & 188 n.213 \\
\hline 4.10 .2 & 83 & 12.8 .2 & $273 ; 274$ n.46 \\
\hline 4.10 .3 & 83 n.43 & 13.14 .3 & 274 \\
\hline 4.16 & 253 & 13.20 .2 & 236 n.6; 242 n.35 \\
\hline 4.21 & 153 n.42 & 13.42 .3 & 274 \\
\hline $4.23-26$ & 253 & 14.2 & 236 \\
\hline 4.29 & 80 n.23 & 14.7.4 & 274 \\
\hline 4.32 & 17 n.33 & 14.11 .3 & 209 n.31 \\
\hline 4.34 & 153 n.42 & 14.19 & 239 \\
\hline $4.34-35$ & $246 ; 251$ & 14.20 .5 & 132 n.41 \\
\hline 4.34 .1 & $246 ; 254$ & 14.23.1 & 228 n.118 \\
\hline 4.41 .2 & 78 n.10 & 14.53 & 170 n.131 \\
\hline 4.42 & 153 n.42 & $14.53-54$ & 274 \\
\hline 4.44 & 80 n.23 & $14.55-56$ & 274 \\
\hline 4.53 & 246 & 15.26 .1 & 228 n.118 \\
\hline 4.53 .2 & $241 ; 242$ n.32 & 15.74 .3 & $243 ; 244$ \\
\hline 4.54 .1 & 82 n. 41 & 16.22 .3 & 243 n.40 \\
\hline 4.57 .3 & 46 & 16.28 .1 & 241 \\
\hline 4.61.1 & 81 n.29 & dial. & \\
\hline $4.70 .1-2$ & 240 & 4 & $152 \mathrm{n} .40$ \\
\hline $4.70 .1-3$ & 127 & $5-6$ & 181 n.183 \\
\hline 5.1 .1 & 84 n.54 & 18 & 170 n.133 \\
\hline 5.2 .1 & 126 n.14 & 23.2 & $238 ; 239$ \\
\hline 5.103 & 245 & 30 & 152 n.38 \\
\hline 6.1 .1 & 126 n.18 & Germ. & \\
\hline 6.3 & 171 n.141 & 33 & 178 n.168 \\
\hline 6.4 .4 & 80 n.26 & hist. & \\
\hline 6.7 .5 & $243 ; 243$ n.39 & 1.1 & 9 n.3; 17 n.33; 98; \\
\hline 6.15 & 169 n.127 & & 147 n.18; 173 n.146; \\
\hline $6.16-17$ & 126 n.14 & & 179 n.172; 184 n.199; \\
\hline 6.16 & 254 & & 251 \\
\hline 6.19 .2 & 127 & 1.1.1 & 103 n.11; 128 n.21; \\
\hline 6.25 .3 & 126 n.15 & & 348 n.70; 348 n.70 \\
\hline $6.26 .1-2$ & 240 & 1.3.1 & 223 n.95 \\
\hline 6.28 & 240 & 1.16 & 176 n.161 \\
\hline 6.29 & 153 n.42 & 2.26 .2 & 209 n.31 \\
\hline 6.29 .3 & 83 n. $46 ; 153$ n.42 & 3.10 .4 & 207 \\
\hline \multirow[t]{2}{*}{6.31 .1} & $140 ; 140$ n.73; 140 & 3.34 & 176 n.161 \\
\hline & n.74 & & \\
\hline 6.32 .2 & 83 n.44 & & \\
\hline 6.40 .1 & 82 n.42 & & \\
\hline 6.51 & 251 & & \\
\hline
\end{tabular}


Terentius Afer, $\mathbf{P}$.

Ad.

332

Hec.

60-62

136 n.60

136 n. 60

Trogus

38.4.1-7.10 200

38.7.4

207

38.4 .15

207

Valerius Maximus

$\begin{array}{ll}1.5 .2 & 117 \mathrm{n} .61 \\ 4.6 .3 & 207\end{array}$

Varro, M. Terentius

Men.

$31 \quad 173$ n.148

De vita populi Romani 4

fr 114 Riposati

135 n. .58

Velleius Paterculus

1.1.1

1.1.4

203

215

1.2.1

1.2 .2

1.3.1

1.3.2

1.7.1

1.8 .1

1.8 .4

1.9.5-6

1.10 .1

1.10 .2

1.11.1

1.11.2

1.11.7

1.12 .3

1.12 .5

1.12 .6

1.13 .5

1.16 .1

1.16 .4

1.17 .1

1.17.5-7

1.17.6

1.17.7
215

215

215

215

216

214 n. 56

214 n. 56

215

226 n.112

216 n. 69

226 n.111; 226 n.112

216 n. 69

223

226 n.111

176 n. $160 ; 216$ n.69

148 n. $18 ; 214$ n.56

214 n. 56

213 n.52; 224 n.102

216 n.69

216

176 n.161

108 n.33

216 n. 69
1.129.3

2.1.1-2

2.1.1

2.1.4

2.1.5

2.2.1

2.2.2-3

2.2.2

2.3.1

2.3.3

2.3.4

2.4 .1

2.4 .6

2.5.1

2.6 .3

2.7.1

2.7.3

2.7.5

2.10

2.13 .2

2.14.1

2.15 .1

2.16 .2

2.18.1-3

2.18 .3

2.18.4-6

2.18 .4

2.18 .5

2.19 .4

2.24 .3

2.26.2

2.27.1-2

2.28 .2

2.29.1

2.29.2

2.32 .1

2.33.1

2.34 .3

2.37 .2

2.38 .1

2.40 .3

2.40 .5

2.41.1-2

2.41 .1

2.41 .3
79 n.17

147 n.18; 166 n.109;

221

106 n.24; 111 n.41;

157 n. 64; 176 n.161

216; 216 n.69

227 n.116

216

226 n.111

$148 \mathrm{n} .18$

226 n.111

148 n. 18

176 n.161

226 n.111; 226 n.114

223

226 n.114

216 n. 69

216 n. 69

216 n. 69

214 n.56

176 n.161

178 n. $168 ; 226$ n.113

216 n. 69

178 n.168; 216 n.69

214 n. 57

226 n.111

178 n.168

226 n.111

178 n.168

216 n. 69

223

226 n.109

223

226 n.111

178 n.168; 216 n.69

216 n. 69; 226 n.111

213 n. 52

224 n.99

226 n.114

226 n.111

216

213 n.52

216

178 n.168; 223

226 n. 111

213 n. 52

178 n.168 


\begin{tabular}{|c|c|c|c|}
\hline 2.42 .3 & 178 n.168 & $2.94 .1-3$ & 226 n.111 \\
\hline 2.42 .4 & 178 n.168 & 2.94 .4 & 226 n.109 \\
\hline 2.43 .1 & 226 n.109 & 2.95 .2 & 226 n.109 \\
\hline \multirow[t]{2}{*}{2.45 .1} & 178 n.168; 216; 226 & 2.96 .2 & 214 n.56 \\
\hline & n.111 & 2.96 .3 & 213 n. 52 \\
\hline 2.47 .2 & 178 n.168 & 2.97 .1 & 224 \\
\hline 2.48 .1 & 178 n.168 & 2.98 .2 & 178 n.168 \\
\hline 2.48 .2 & 178 n.168 & 2.99.1-2 & 226 n.109 \\
\hline 2.48 .3 & 216 & 2.99 .3 & 213 n.52 \\
\hline 2.48 .5 & 213 n.52 & 2.99 .4 & 178 n.168; 213 n.52 \\
\hline 2.49 .1 & 214 n.56 & 2.100 .4 & 178 n.168 \\
\hline 2.49 .3 & 223 & 2.101 .3 & 214 n.56 \\
\hline $2.49 .4-50.2$ & 227 & 2.102 .3 & 178 n.168 \\
\hline $2.51 .1-2$ & 226 n.109 & 2.103 .1 & 214 n.56 \\
\hline 2.52 .3 & 213 n.52 & 2.103 .4 & 213 n. 52 \\
\hline 2.54 .3 & 216 n.69 & 2.104 .2 & 214 n.56 \\
\hline 2.55 .1 & 213 n.52; 216 & 2.104 .3 & 214 n.57 \\
\hline 2.55 .3 & 216 n.69 & 2.108 .2 & 213 n.52 \\
\hline 2.56 .3 & 226 n.109 & 2.109 .1 & 216 \\
\hline 2.57 .3 & 178 n.168 & 2.111 .4 & 218 \\
\hline 2.58 .2 & 178 n.168 & 2.112 .2 & 226 n.109 \\
\hline 2.59 .6 & 216 n.69 & 2.112 .4 & 178 n.168 \\
\hline 2.61 .2 & 216 n.69 & 2.112 .7 & 248 \\
\hline $2.62 .1-3$ & 226 n.109 & 2.113 .1 & 214 n.56 \\
\hline 2.63 .1 & 178 n.168 & 2.113 .2 & 226 n.109 \\
\hline 2.65 .2 & 214 n.56 & 2.114 .4 & 213 n. 52 \\
\hline 2.66 .3 & 178 n.168; 213 n.52 & 2.115 .1 & 214 n.57 \\
\hline 2.68 .1 & 178 n.168; 226 n.111 & 2.116 .3 & 178 n.168 \\
\hline $2.69 .1-5$ & 233 n.131 & 2.119 .1 & 213 n.52 \\
\hline $2.69 .3-4$ & 178 n.168 & 2.119 .2 & 226 n.109 \\
\hline 2.69 .5 & 214 n.57 & 2.120 .2 & 216 n.69 \\
\hline 2.70 .2 & 226 n.109 & 2.121 .3 & 214 n.57 \\
\hline 2.75 .2 & 178 n.168 & 2.123 .2 & 226 n.109 \\
\hline 2.75 .3 & 226 n.111 & 2.124 .1 & 213 n. 52 \\
\hline 2.76 .1 & 226 n.111; 214 n.57 & 2.124 .3 & 248 \\
\hline $2.83 .1-2$ & 226 n.111 & 2.124 .4 & 214 n.57 \\
\hline 2.83 .1 & 178 n.168 & 2.125 .1 & 216 n.69 \\
\hline 2.85 .3 & 223 & $2.126 .1-5$ & 214 \\
\hline 2.86 .1 & 213 n.52 & 2.127 .1 & 216 n.69 \\
\hline 2.87 .1 & 216 & 2.127 .3 & 216 n.69 \\
\hline $2.88 .2-3$ & 248 & $2.129 .1-130.5$ & 214 \\
\hline 2.88 .2 & 178 n.168 & 2.129 .3 & 216 n.69 \\
\hline 2.89 .1 & 213 n.52 & 2.130 .4 & 214 n.56 \\
\hline $2.91 .2-3$ & 226 n.111 & 2.131 .1 & 168 n.121 \\
\hline 2.91 .2 & 178 n.168 & & \\
\hline 2.91 .3 & 226 n.111 & & \\
\hline 2.92 .2 & 226 n.111 & & \\
\hline
\end{tabular}


Vergilius Maro, $\mathbf{P}$.

Aen.

1.373

4.178

4.489

5. 295

8.90

9.539
12 n.12

205 n.19

175 n.156

167 n. 118

209 n. 31

175 n.156 georg.

1.199-200

175 n.156

2.200

175 n.156

4.495

175 n.156

Victorinus, Marius

rhet. Cic. 158 Halm 147 n.18 